\title{
Amyloid- $\beta$ peptide dimers undergo a random coil to $\beta$-sheet transition in the aqueous phase but not at the neuronal membrane
}

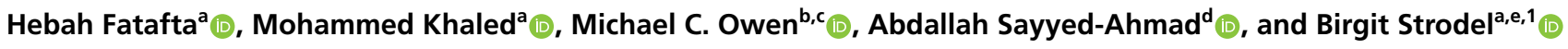 \\ ${ }^{a}$ Institute of Biological Information Processing (IBI-7: Structural Biochemistry), Forschungszentrum Jülich, 52425 Jülich, Germany; ${ }^{b}$ Central European \\ Institute of Technology, Masaryk University, Brno 625 00, Czech Republic; 'Institute of Chemistry, University of Miskolc, 3515 Miskolc-Egyetemváros, \\ Hungary; ${ }^{d}$ Department of Physics, Birzeit University, 71939 Birzeit, Palestine; and eInstitute of Theoretical and Computational Chemistry, Heinrich Heine \\ University Düsseldorf, 40225 Düsseldorf, Germany
}

Edited by Gerhard Hummer, Max Planck Institute for Biophysics, Frankfurt am Main, Germany, and accepted by Editorial Board Member Angela M. Gronenborn August 16, 2021 (received for review March 31, 2021)

\begin{abstract}
Mounting evidence suggests that the neuronal cell membrane is the main site of oligomer-mediated neuronal toxicity of amyloid- $\beta$ peptides in Alzheimer's disease. To gain a detailed understanding of the mutual interference of amyloid- $\beta$ oligomers and the neuronal membrane, we carried out microseconds of all-atom molecular dynamics (MD) simulations on the dimerization of amyloid- $\beta$ $(A \beta) 42$ in the aqueous phase and in the presence of a lipid bilayer mimicking the in vivo composition of neuronal membranes. The dimerization in solution is characterized by a random coil to $\beta$ sheet transition that seems on pathway to amyloid aggregation, while the interactions with the neuronal membrane decrease the order of the $A \beta 42$ dimer by attenuating its propensity to form a $\beta$-sheet structure. The main lipid interaction partners of $A \beta 42$ are the surface-exposed sugar groups of the gangliosides GM1. As the neurotoxic activity of amyloid oligomers increases with oligomer order, these results suggest that GM1 is neuroprotective against A $\beta$-mediated toxicity.
\end{abstract}

Alzheimer's disease | amyloid- $\beta$ | neuronal membrane | molecular dynamics | transition network

n Alzheimer's disease (AD), amyloid- $\beta$ peptide $(\mathrm{A} \beta)$ aggregates into fibrils and subsequently accumulates as plaques within the neural tissue (1). An increasing number of studies suggest that the smaller soluble oligomers formed in the earlier stages of the aggregation process are the main cytotoxic species affecting the severity and progression of $\mathrm{AD}(2-4)$. A $\beta$ dimers have been reported to be the smallest toxic oligomer that affects synaptic plasticity and impairs memory $(5,6)$. Therefore, a detailed characterization of $\mathrm{A} \beta$ dimerization is an essential step toward developing a better understanding of the aggregation process. However, its transient nature (resulting from its high aggregation tendency), its plasticity, and its equilibrium with both the monomer and higher-order oligomers all make the $\mathrm{A} \beta$ dimer extremely challenging to study experimentally. In fact, a large amount of the experimental studies performed on $\mathrm{A} \beta$ dimers employ some kind of cross-linking to stabilize them (7-9). On the other hand, covalently cross-linked $\mathrm{A} \beta$ dimers are certainly of biological relevance, as such species have been retrieved from the brains of $\mathrm{AD}$ patients and their neurotoxicity has been demonstrated $(6,10)$. Apart from this, recent technological developments, such as advanced single-molecule fluorescence spectroscopy and imaging, opened the way to characterize amyloid oligomers without the need to stabilize them by cross-linking $(11,12)$. Molecular dynamics (MD) simulations are also able to provide atomic insight into the temporal evolution of the dimer structure without the need of cross-linking $(13,14)$. Previous simulations of $\mathrm{A} \beta$ dimers were modeled in the aqueous phase only, and thus they lacked essential details from the cellular context. Consideration of the latter is particularly important if one wishes to reveal the mechanism of toxicity that has been shown to rely on direct contact with the lipid membrane of neurons by $\mathrm{A} \beta$ oligomers $(15,16)$.

Many studies have been done to understand the consequences of $\mathrm{A} \beta$-membrane interactions; however, it is extremely difficult to capture these transient interactions with experimental methods. This becomes possible with MD simulations and this problem is addressed in the current work. We use an aggregate of $24 \mu$ s of MD simulations to investigate the dimerization of the full-length $\mathrm{A} \beta 42$ peptide both in solution and in the presence of a model lipid bilayer including six lipid types to mimic the composition of a neuronal cell membrane (17-19): 38\% 1-palmitoyl-2-oleoyl-sn-glycero-3phosphocholine (POPC), 24\% 1-palmitoyl-2-oleoyl-sn-glycero3-phosphoethanolamine (POPE), 5\% 1-palmitoyl-2-oleoyl-snglycero-3-phospho-L-serine (POPS), 20\% cholesterol (CHOL), $9 \%$ sphingomyelin (SM), and $4 \%$ monosialotetrahexosylganglioside (GM1) (Fig. 1A). For modeling $\mathrm{A} \beta$ we employ Charmm $36 \mathrm{~m}$, a force field adjusted for intrinsically disordered proteins (IDPs), to model their preference to adopt extended structures. When applied to monomeric $\mathrm{A} \beta$, Charmm $36 \mathrm{~m}$ yields more than $80 \%$ of the structures in a random coil and extended

\section{Significance}

The aggregation of the amyloid- $\beta$ peptide $(A \beta)$ into neurotoxic oligomers is central to the development of Alzheimer's disease. One possible source of their toxicity results from interactions of the $A \beta$ oligomers with the neuronal membrane, damaging membrane integrity and thus neurons. However, molecular details of these interactions are unclear. Here, we contrast the dimerization of $A \beta$ in solution and at the neuronal membrane. Our results clearly indicate that the sugar moieties of GM1 sequester $A \beta$ by forming key hydrogen bonds with the peptide, which diverts the configuration of the $A \beta$ dimers away from damaging $\beta$-sheet-rich structures. These findings underline the importance of GM1 in Alzheimer's disease progression and provide a nanoscopic basis for its reported neuroprotective effect.

Author contributions: H.F., M.C.O., and B.S. designed research; H.F., M.K., and M.C.O performed research; H.F., M.K., and M.C.O. analyzed data; H.F., A.S.-A., and B.S. wrote the paper; and A.S.-A. and B.S. supervised the work.

The authors declare no competing interest.

This article is a PNAS Direct Submission. G.H. is a guest editor invited by the Editorial Board.

This open access article is distributed under Creative Commons Attribution-NonCommercialNoDerivatives License 4.0 (CC BY-NC-ND).

${ }^{1}$ To whom correspondence may be addressed. Email: b.strodel@fz-juelich.de.

This article contains supporting information online at https://www.pnas.org/lookup/suppl/ doi:10.1073/pnas.2106210118/-/DCSupplemental.

Published September 20, 2021 

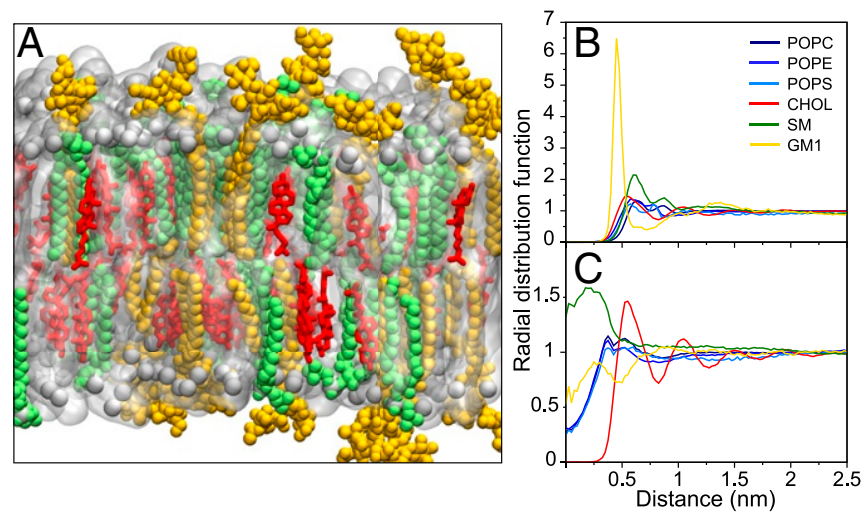

Fig. 1. (A) A snapshot of the neuronal membrane containing $38 \%$ POPC, $24 \%$ POPE, 5\% POPS (collectively shown as gray surface with their phosphorous atoms indicated by gray spheres), $20 \% \mathrm{CHOL}$ (red sticks), 9\% SM (green spheres), and 4\% GM1 (yellow spheres). In the following, PC, PE, and PS are synonymously used for POPC, POPE, and POPS, respectively. ( $B$ and $C$ ) Radial distribution functions for $(B)$ lipid pairings of identical type and $(C)$ lipidCHOL pairings. The $\mathrm{P}$ atoms of $\mathrm{PC}, \mathrm{PE}, \mathrm{PS}$, and $\mathrm{SM}$ and the $\mathrm{O}$ atoms of $\mathrm{CHOL}$ and GM1 were used as reference atoms for the RDF calculations. The RDFs are averaged over both membrane leaflets. The $x$ axis shows the distances between the respective atom pairs. Since $\mathrm{CHOL}$ resides deeper inside the membrane, it is possible that the $\mathrm{O}$ atom of $\mathrm{CHOL}$ and the reference atoms of the other lipids are above each other, explaining why not all of the RDFs approach zero for $x=0$. The colors of the functions refer to the lipids as indicated in the color key in $B$. Pairs with RDF $>1$ are considered to form clusters.

state, and the remaining ones feature transient $\beta$-hairpins, which is in acceptable agreement with experimental data (20). Moreover, Charmm $36 \mathrm{~m}$ outperforms other force fields when it comes to modeling peptide aggregation $(21,22)$. To the best of our knowledge, this simulation study breaks ground on two fronts: 1) It exceeds the simulation time of previous studies modeling $\mathrm{A} \beta$-membrane interactions by an order of magnitude, and 2) it studies the aggregation of $\mathrm{A} \beta$ on a bilayer containing more than three different lipid types. Lipid bilayers of a complexity comparable to the one modeled here have been thus far studied only at the coarse-grained level $(23,24)$. We also analyze the aggregation pathways by transition networks (25-27), which elucidate the similarities and differences between $\mathrm{A} \beta$ dimerization steps both in solution and at the neuronal membrane. We find that the neuronal membrane reduces the dynamics of membrane-bound $\mathrm{A} \beta 42$ while it also inhibits $\beta$-sheet formation. Here, the sugar groups of GM1 form hydrogen bonds with the peptide, thereby reducing the possibilities for other hydrogen bonds to otherwise form. In contrast, the dimerization in the aqueous phase is characterized by a random coil to $\beta$ sheet transition, leading to $\beta$-sheet structures similar to the ones found in $\mathrm{A} \beta$ fibrils.

\section{Results}

The Neuronal Membrane Is in a Liquid Ordered Phase. Before we analyze the interaction of $\mathrm{A} \beta$ with the neuronal membrane, we determine the characteristics of the latter. The mass density profile of each lipid and water along the membrane $z$ axis (SI Appendix, Fig. S1) shows the distribution of the bilayer components, as well as the bilayer thickness. The positions of the headgroups are at similar locations for POPC (PC), POPE (PE), POPS (PS), and SM. CHOL, on the other hand, is shifted toward the hydrophobic core of the bilayer, while GM1 is farther away from the bilayer center, due to the protrusion of the sugar groups from the $x y$ surface of the membrane (Fig. $1 A$ ). The headgroupto-headgroup distance of PC, PE, and PS indicates a bilayer thickness of $4.65 \pm 0.03 \mathrm{~nm}$.
We calculated the acyl chain order parameter $S_{\mathrm{CH}}$ of the C-H bonds of all the lipid tails (SI Appendix, Fig. S2) to gain insight into their arrangement within the membrane. Values of 0.35 to 0.4 for the order parameters of carbon atoms 4 to 10 are reached, which is an increase compared to the order parameters found in other membranes $(28,29)$. This is due to the effects of cholesterol and sphingomyelin, which are known for their role in increasing lipid order. Notably, we find the acyl chains of GM1 and SM to be the most ordered. We can thus conclude that the neuronal membrane is in the liquid-ordered state, which is in agreement with previous observations $(24,30)$.

GM1 Forms Ganglioside Clusters. The radial distribution function (RDF) of all possible lipid pairings was calculated to monitor the effect of these pairwise interactions on lipid clustering (Fig. $1 B$ and $C$ and SI Appendix, Fig. S3). A distinct RDF peak is seen at $\approx 0.45 \mathrm{~nm}$ for the self-clustering of GM1 and pronounced peaks are seen at 0.55 and $0.6 \mathrm{~nm}$ for the formation of CHOL and SM clusters, respectively, while all other lipids do not tend to self-associate. The self-clustering of GM1 is considerably stronger than that of the other lipids. Thus, taking the relatively low concentration of GM1 (4\%) into account, one can conclude that GM1 has a strong tendency to selfassociate that can result in its sorting. No strong clustering between mixed lipid pairs is observed. Notable coassociation is seen only for SM with POPE, CHOL, and GM1. Interestingly, the RDF of PE-PS has a higher peak compared to that of PE-PE and PS-PS, respectively. The dispersion of PS is understandable given that it is negatively charged. The negative charge of both GM1 and PS also explains why these two lipids avoid coclustering.

To elucidate the dominant lipid-lipid interactions underlying the RDFs, the average numbers of hydrogen bonds ( $\mathrm{H}$ bonds) between the different lipid pairs were evaluated. SI Appendix, Fig. S4 shows that the sorting of GM1 results from its ability to build a network of $\mathrm{H}$ bonds via its sugar headgroups, despite its negative charge. The propensity of SM to form $\mathrm{H}$ bonds with itself also gives rise to its self-clustering, whereas the minor self-clustering seen for CHOL is a result of the cholesterol condensing effect. This effect does not result from attractive van der Waals interactions between CHOL molecules, but from a reduced membrane perturbation energy if small cholesterol domains are formed (31). However, such cholesterol clusters are not particularly stable, as evidenced by only a small peak in the RDF for CHOL-CHOL. The coclustering of CHOL and $\mathrm{SM}$ is facilitated by $\mathrm{H}$ bonds formed between the hydroxyl of CHOL and the amide group of SM, which agrees with previous findings (32). The RDF profile of SM-GM1 can also be explained by $\mathrm{H}$-bond formation. We conclude that $\mathrm{H}$ bonds play an essential role in stabilizing lipid clusters within the neuronal membrane.

A $\beta 42$ Dimerizes at the Neuronal Membrane and Interacts with GM1. To understand the effects of the neuronal membrane on the aggregation of $\mathrm{A} \beta 42$, we analyzed the $6 \times 2 \mu$ s of MD data in the presence of the lipid bilayer and compared the aggregation to the $6 \times 2 \mu$ s of MD simulations done in the aqueous phase. We first assess whether and how the two peptides bind to and interact with the membrane.

To follow the association between $\mathrm{A} \beta 42$ and the neuronal membrane, we calculated the minimum distance of both peptides from the lipid bilayer surface for each of the six simulations (SI Appendix, Fig. S5). It can be seen that peptide 1 usually interacts with the membrane at a closer distance than peptide 2 does, which can be explained by the fact that the initial structures of five of the six simulations were selected from the initial $2-\mu$ s simulation. This allows us to better elucidate the effects of the membrane on the preferentially membrane-bound 
peptide as their mutual interaction time is larger than it would have been if both peptides had the same interaction probability. Nonetheless, both peptides tend to be associated with the membrane as an intact dimer, since if one peptide is $>0.5 \mathrm{~nm}$ away from the membrane, implying that this peptide is in solution, then very often this is also the case for the other peptide. Fig. 2 shows representative snapshots for the membrane association of $\mathrm{A} \beta 42$, including one for loose binding in Fig. $2 A$. Fig. $2 B$ represents the situation where peptide 1 is in close contact with the membrane, while peptide 2 is a bit farther away. The opposite, less prevalent situation with peptide 2 being closer is depicted in Fig. $2 C$ and is less common, while Fig. $2 D$ shows how both peptides can bind tightly to the membrane. Fig. 2 further suggests that $A \beta 42$ tends to interact with GM1 instead of the other lipids and that $\beta$-sheets are the dominating secondary structure in peptide 2 but not in the more membrane-bound peptide 1 . The analysis of the contacts between $\mathrm{A} \beta 42$ and the various lipids confirms that the peptide has a high tendency to associate with GM1, followed by PC, PE, and PS (SI Appendix, Fig. S6). Here, we emphasize that these contacts are not normalized but absolute values. Considering that only $4 \%$ of the lipids are GM1 while the phospholipids make up for more than two-thirds of the membrane, one can thus conclude that $\mathrm{A} \beta 42$ is highly attracted to GM1. Interestingly, almost no contacts are made with CHOL or SM.

To rationalize the driving force that controls $\mathrm{A} \beta 42$ interaction with the membrane surface, the interaction energy of each A $\beta 42$ residue with each of the lipid components was calculated and partitioned into its electrostatic $\left(E_{\mathrm{Coul}}\right)$ and Lennard-Jones $\left(E_{\mathrm{LJ}}\right)$ contributions (Fig. 3). Notably, the lipid interactions of peptide 1 are more favorable than those of peptide 2, agreeing with the observation that peptide 1 interacted more strongly with the membrane. Our results suggest that the major driving force for the association of the peptides to the membrane is the electrostatic attraction to PC, PE, and PS, especially via the highly charged N-terminal region and residues F20 to A30. Residues at the $\mathrm{N}$ terminus had the strongest interactions with the membrane, such as D1, E3, and D7 with PE; D1 with PC; and R5 with PS. The latter interaction involves $\mathrm{H}$-bond formation (SI Appendix, Fig. S7), which is enabled via the carboxylate group of $\mathrm{PS}$, whereas the primary ammonium group of $\mathrm{PE}$ forms $\mathrm{H}$ bonds with D1, E3, and D7. The tertiary ammonium group of PC, on the other hand, does not support H-bond formation, leading to a relatively low $\mathrm{H}$-bond propensity between PC (via its phosphate group) and $\mathrm{A} \beta 42$. The interactions between $\mathrm{GM} 1$ and $\mathrm{A} \beta 42$ are driven by both Coulomb and Lennard-Jones energies (Fig. 3) and are facilitated by the sugar headgroups of GM1, which protrude from the membrane and are therefore particularly accessible to A $\beta 42$. Moreover, the interactions with GM1 derive from a considerable number of $\mathrm{H}$ bonds, which involve almost all residues of both peptides, but particularly those of peptide 1 .

No direct interaction between $\mathrm{A} \beta 42$ and $\mathrm{CHOL}$ was observed (SI Appendix, Fig. S6), due to the deeper, unexposed position of CHOL within the membrane. Interestingly, even though SM has the same headgroup as PC, which is also located at a similar position along the bilayer normal, $\mathrm{A} \beta 42$ hardly interacted with SM. This can be understood by considering the preference of SM to form $\mathrm{H}$ bonds with other lipids including itself, which reduces its tendency to create $\mathrm{H}$ bonds with the peptide.

The A $\beta 42$ Dimer Does Not Affect the Neuronal Membrane. To determine whether the peptides affect the structure of the lipid membrane, we calculated the lipid order parameter for the lipids that are within $0.5 \mathrm{~nm}$ of the peptide when adsorbed to the membrane (SI Appendix, Fig. S2). The results suggest no notable change in the lipid order parameter due to the interactions with A $\beta 42$. Moreover, only a slight deviation of about $\pm 0.1 \mathrm{~nm}$ was seen in the bilayer thickness (SI Appendix, Fig. S8) at the site of peptide interaction. We thus conclude that the peptides interact only with the lipid headgroups without inserting into the membrane, thereby preventing larger changes in the membrane order and thickness.

Different Aggregation Pathways in Solution and at the Neuronal Membrane. To unravel differences within the aggregation pathways, we computed transition networks (TNs) for the $\mathrm{A} \beta 42$ dimerization both in the aqueous phase and in the presence of the neuronal membrane. To this end, we characterized the conformations by assigning the aggregate state (monomer or dimer), the number of hydrophobic contacts between the peptides in a dimer, and the number of residues in $\beta$-strand conformation as descriptors. To further simplify the TNs, we grouped both the number of hydrophobic contacts and the number of residues in $\beta$-strand conformation in blocks of five such that we end up with ranges h1 to h12 and b1 to b6. For example, h1 and b1 stand for hydrophobic contacts and the number of residues in $\beta$-strand conformation, respectively, ranging from 1 to 5 . The maximum state h12 involves between 56 and 60 hydrophobic contacts and the b6 state means that between 26 and 30 residues per peptide adopted a $\beta$-strand conformation.

The resulting TNs (Fig. 4) are characterized by two regions: the monomeric region (on the left side of the TNs) and the dimeric region (in the middle and the right side of the TNs), where the former evolves into the latter. These regions are

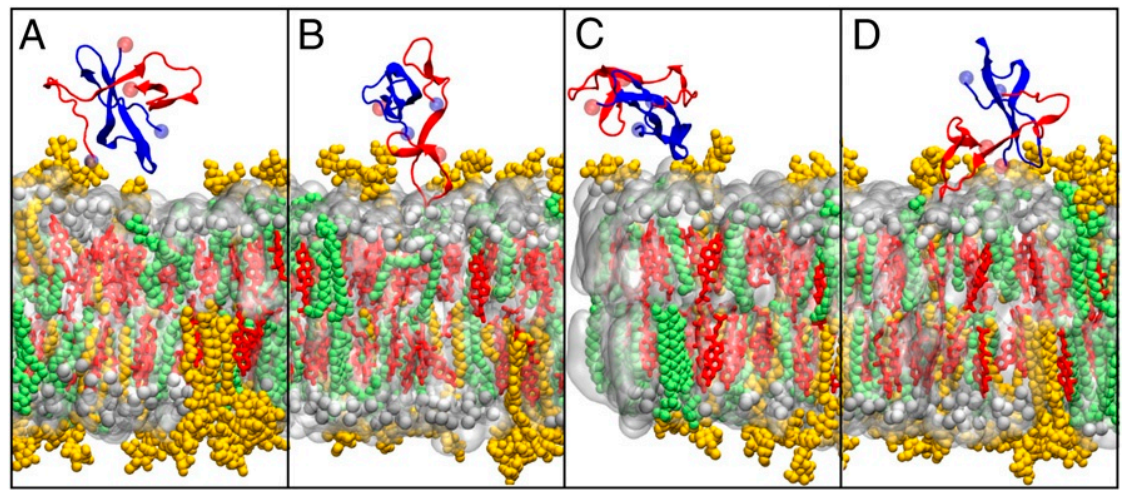

Fig. 2. Snapshots of $A \beta 42$ interacting with the neuronal membrane. Peptide 1 and peptide 2 are shown as cartoons in red and blue, respectively, with their termini indicated by spheres ( $\mathrm{N}$, light blue; $\mathrm{C}$, light red). The color coding for the membrane is the same as in Fig. $1 A$. Representative interaction patterns are provided: $(A)$ both peptides being loosely attached to the bilayer surface, $(B)$ peptide 1 being in close interaction with the membrane and peptide 2 being bound to peptide $1,(C)$ the opposite situation to that in $B$, and $(D)$ both peptides being in close contact with the membrane. 

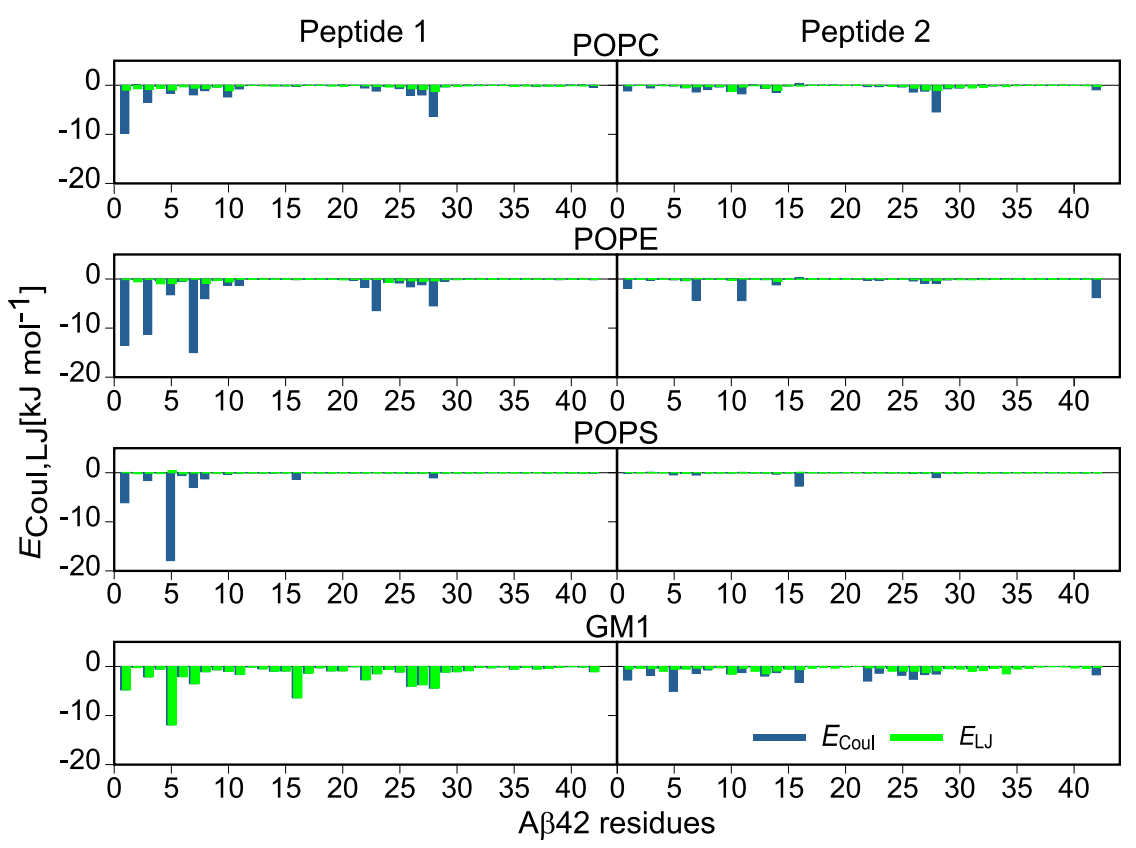

Fig. 3. The average interaction energies of peptide 1 (Left) and peptide 2 (Right) with each lipid of the neuronal membrane. Electrostatic and Lennard-Jones energies are shown in blue and green, respectively. The more negative an energy is, the more attractive is the corresponding interaction.

connected by several bridging nodes, which, on average, are characterized by a higher amount of $\beta$-sheet (i.e., larger $n$ in the descriptor $\mathrm{b} n$ ) in the case of the solution system. In both TNs, a representative bridging node is indicated by a green circle, [2, h2, b6] for the solution system and [2, h2, b2] for the membrane system, which are further augmented by a characteristic structure. In solution, there are more transitions between monomers and dimers, which indicates a higher number of association and dissociation events. In general, the $\mathrm{TN}$ for the solution system exhibits more nodes and transitions.

A closer inspection of both TNs reveals how the two peptides evolve from the monomeric random coil state, which is represented by node $[1,0,0]$ with no interpeptide hydrophobic contacts and no residues in $\beta$-strand conformation, to dimers with only a few hydrophobic contacts, as present in states $[2, \mathrm{~h} 1$, $\mathrm{b} n]$. Here $\mathrm{b} n$ ranges from $\mathrm{b} 1$ to $\mathrm{b} 6$, indicating an increase in $\beta$ strand content as the structure changes along the path through nodes $[1,0, \mathrm{~b} n]$ and $[2,0, \mathrm{~b} n]$. The dimers with no hydrophobic contacts are so-called encounter complexes, where the minimal distance between the two monomers fell below $4.5 \mathrm{~nm}$, and subsequently form stable dimers by increasing their contact area as interpeptide contacts form. This process stabilizes the dimer and is accompanied by an increase in $\beta$-strand content. In solution, the dimers form more interpeptide hydrophobic contacts, reaching states $[2, \mathrm{~h} 12, \mathrm{~b} 6]$ and $[2, \mathrm{~h} 13, \mathrm{~b} 5]$ wherein 50 to $70 \%$ of all A $\beta 42$ residues form a $\beta$-sheet. In the presence of the neuronal membrane, both the hydrophobic contact area and $\beta$-sheet content are reduced, with the maximal values being $[2, \mathrm{~h} 10, \mathrm{~b} 4]$ and $[2, \mathrm{~h} 9, \mathrm{~b} 5]$, explaining the smaller number of nodes in this TN. Some of the interpeptide contacts are replaced by peptide-lipid contacts, which in turn inhibits $\beta$-sheet formation. This conclusion is confirmed by the representative structures shown in Fig. 4 and those illustrating the membrane adsorption of the dimer (Fig. 2). The membrane-adsorbed dimer structures are more compact than the dimer structures in solution, which feature extended $\beta$-sheets.

Long $\beta$-Strands in Solution and Compact $A \beta 42$ Structures at the Membrane. To quantify the effect of both aggregation and membrane adsorption on the peptide secondary structure, we deter- mined the propensity of each residue to adopt a helical conformation, to be part of a $\beta$-sheet, or to be in a turn or bend conformation (Fig. 5A). For the dimer both in solution and on the membrane, $\beta$-sheet formation is observed. Using the same force field, mostly disordered conformations were sampled for the $\mathrm{A} \beta$ monomer during a 30- $\mu$ s MD simulation, with an average $\beta$-sheet content of about $15 \%$ (20). This rises to $36 \%$ for the dimer in solution, which indicates that dimerization causes $\mathrm{A} \beta 42$ to undergo a disorder-to-order transition with $\beta$-sheet folding. The $\beta$-sheet content for the membrane-adsorbed dimer is $28 \%$ and thus smaller compared to that for the solvated dimer. This decrease is particularly pronounced for peptide 1 , which interacts more strongly with the membrane than peptide 2 does. Instead, peptide 1 exhibits more turns, bends, and random coil structures, which suggests that the membrane inhibits $\beta$-sheet formation. Also, no pronounced helix formation is observed for the membrane-bound dimer, which one might expect based on NMR results (33) and previous simulation studies of $\mathrm{A} \beta$ that employed implicit membrane models $(34,35)$. However, a closer inspection of these studies reveals that for helices to be present, the affected $\mathrm{A} \beta$ residues need to be inserted into the hydrophobic membrane core, which did not occur here. It remains to be shown what comes first: helix formation or membrane insertion. In solution, both peptides feature a very similar secondary structure pattern along their primary structure. They display a particularly high propensity for a $\beta$-sheet in the regions Q15 to F20 of the central hydrophobic core $(\mathrm{CHC})$ and $\mathrm{A} 30$ to $\mathrm{V} 40$ from the $\mathrm{C}$-terminal hydrophobic region. This excludes the residue pair G37/G38, which has a tendency to form a turn as previously shown in simulations (36) and NMR spectroscopy (37).

The analysis of the intrapeptide contacts, derived from interresidue distances (Fig. 6), indicates that in solution long $\beta$ hairpins between two antiparallel strands involving residues Y10 to V24 and Q27 to V40 formed in both peptides. These $\beta$ hairpins are particularly stable since the $\beta$-sheet propensity of the strongly hydrophobic regions ${ }^{18} \mathrm{VFF}^{20}$ and ${ }^{32} \mathrm{IGL}^{34}$ even reaches values above $90 \%$. In previous simulation studies, $\beta$ sheet formation upon $\mathrm{A} \beta$ dimerization has also been the prevailing finding (see table 2 of ref. 36 and references therein 


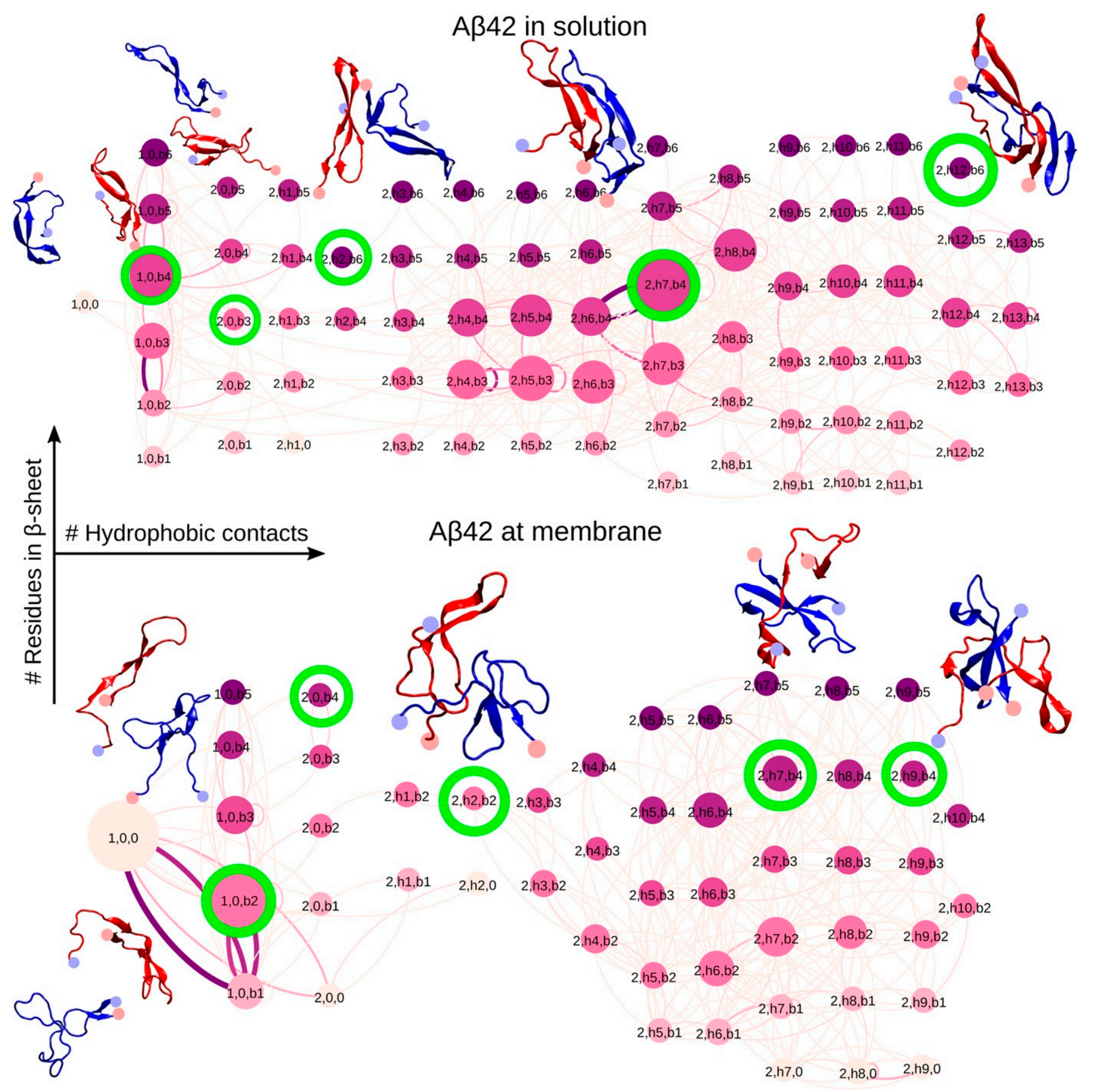

Fig. 4. The TN for $A \beta 42$ dimerization in the aqueous phase (Top) and in the presence of the neuronal membrane (Bottom). Each node is defined by three descriptors: oligomer size, number of interpeptide hydrophobic contacts, and number of residues in $\beta$-strand conformation. The last two descriptors are grouped in blocks of five and are named h1 to h12 for hydrophobic contacts and b1 to b6 for the number of residues in $\beta$-strand conformation. The nodes are connected by edges that represent transitions between the connected peptide states. The size of the nodes and the thickness of the edges are proportional to the respective state or transition probability. They are colored based on the descriptor reflecting the number of residues in $\beta$-strand conformation (from light pink for no $\beta$-sheets to dark purple for the maximum amount of $\beta$-sheets in b6). For the nodes circled in green representative peptide conformations are shown (see color code in Fig. 2).

as well as refs. $38-41)$. As found here, the $\beta$-sheets are preferentially formed between the C-terminal hydrophobic regions, followed by the involvement of the CHC. However, in most of these previous studies, the $\beta$-sheets are shorter and the overall dimer appearance is more compact. This likely resulted from the usage of older force fields, which were not optimized for IDPs and are known to provide too compact IDP conformations (42). Exceptions are a coarse-grained discrete MD study
(38) and a structure-prediction study for transmembrane $\mathrm{A} \beta$ oligomers (35) that yielded similarly extended $\beta$-sheets. This is confirmed by comparing the intrapeptide contacts that are present in the different A $\beta 42$ dimers, as shown in SI Appendix, Fig. S9. SI Appendix, Fig. S9 further shows that the $\beta$-hairpin centered at G25/S26 coincides with the peptide regions that are involved in the cross- $\beta$-sheet structure found in U-shaped $\mathrm{A} \beta$ fibrils $(43,44)$. 


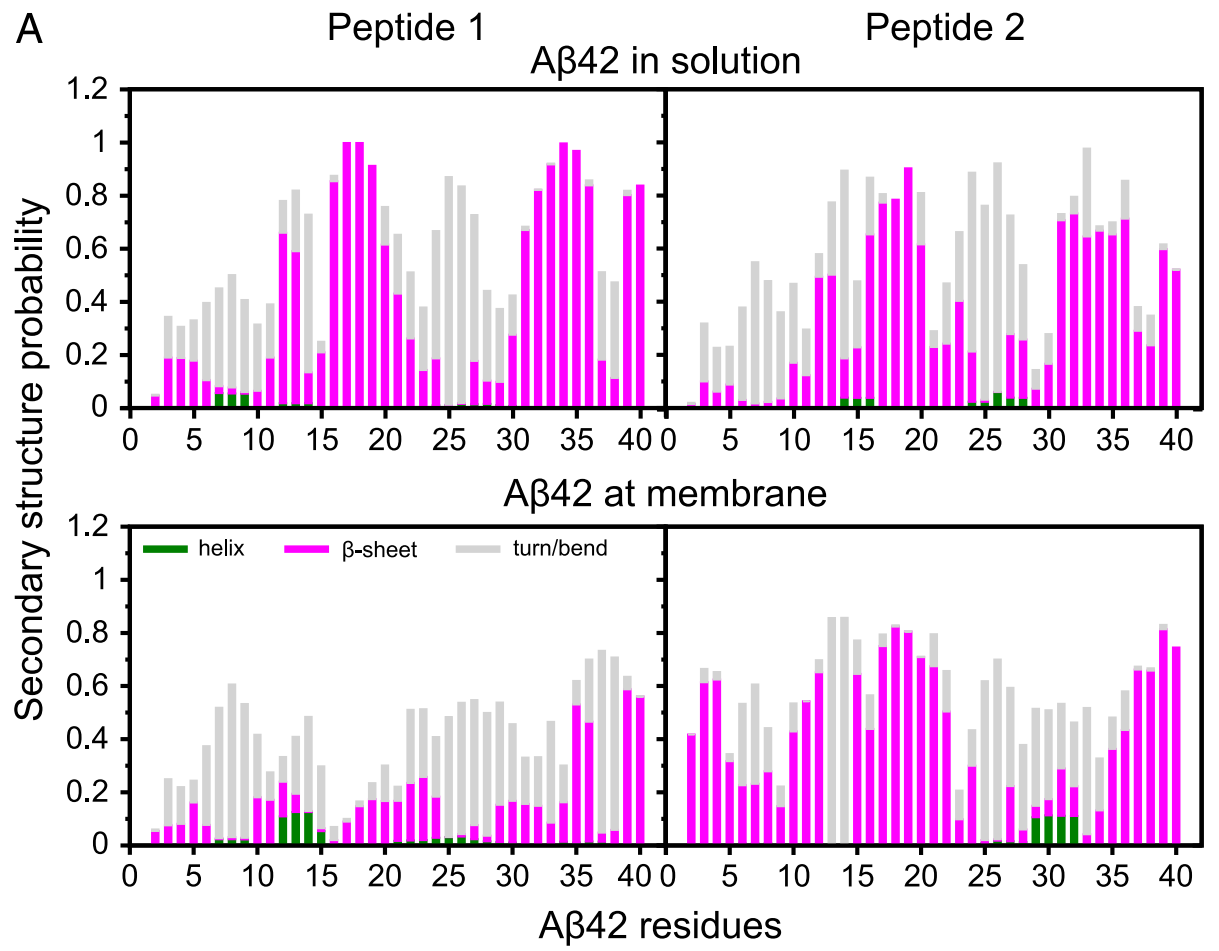

B
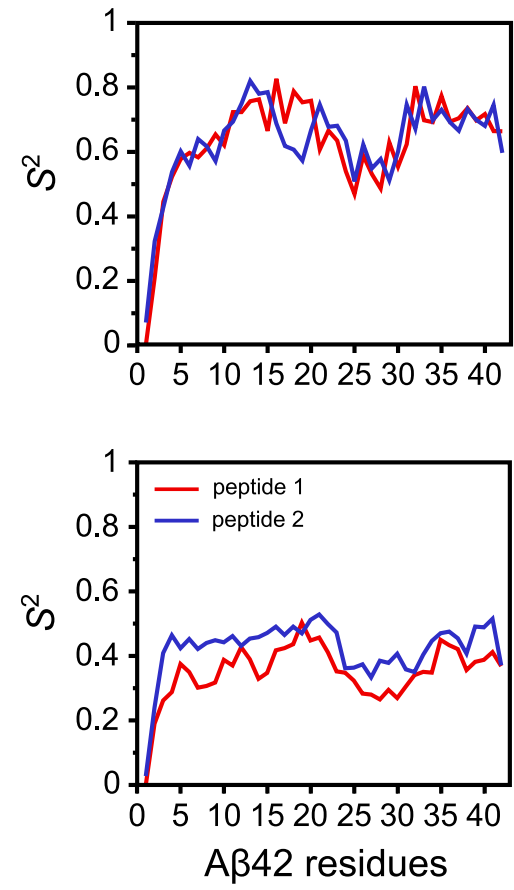

Fig. 5. Structural characteristics of the dimer in the aqueous phase (Top) and in the presence of the neuronal membrane (Bottom). ( $A$ ) Probability of secondary structures to form in each residue of the peptides. The bars represent the cumulative secondary structure probabilities consisting of helix (green), $\beta$-strand/bridge (magenta), and turn or bend (gray). The difference from 1.0 presents the probability of the random coil state. ( $B$ ) The average order parameter $S^{2}$ of each residue and peptide.

The intrapeptide contacts present in the membrane-adsorbed dimer are more diverse and different in the two peptides. For peptide 2 they reveal the prevalence of two shorter hairpins, one centered at $\mathrm{H} 14$ and the other one at G25, and several contacts between $\mathrm{N}$ - and $\mathrm{C}$-terminal residues. The more membrane-adsorbed peptide 1 , on the other hand, is devoid of noteworthy contacts involving its $\mathrm{N}$-terminal residues. These are the amino acids that preferentially interact with the membrane and are therefore not available for interresidue interactions. In the $\mathrm{C}$-terminal region of peptide 2 the formation of a very short $\beta$-hairpin is visible. Overall, the intrapeptide contacts corroborate the conclusion that at the membrane $\mathrm{A} \beta 42$ adopts more compact conformations with less $\beta$-sheet than the dimer in solution.

Dimerization in Solution Is Mainly Driven via the Hydrophobic CTerminal Region. To obtain an overview of how the two peptides are arranged with respect to each other as dimers, we calculated the interpeptide distances on a per-residue basis. The resulting distance matrices for the two dimer systems (Fig. 6) are almost symmetric with respect to their diagonal and are characterized by areas of high contact density along the diagonal as well as in the upper left and lower right quadrants. Only the D23 to K28 region in both peptides and in both environments does not show a noteworthy contact propensity. This is the same region of the peptide that we assigned a turn or bend conformation (Fig. 5). It can thus be concluded that this bend/turn region does not form the interpeptide interface.

For the dimer in solution, the highest contact density is observed between the two C-terminal regions, A30 to A42, which are the same regions where a high $\beta$-propensity was identified. Therefore, these two $C$-terminal regions not only are involved in intrapeptide $\beta$-sheets, but also form an interpeptide $\beta$-sheet in solution. This is confirmed when analyzing the residue-residue interaction energies between the two peptides, which involve
Coulomb interactions deriving from backbone $\mathrm{H}$ bonds and Lennard-Jones energies originating from interactions between hydrophobic residues (SI Appendix, Fig. S10). While the distance matrix does not show a clear preference for either an antiparallel or a parallel $\beta$-sheet between the two C-terminal regions, and both arrangements are indeed possible (see the representative conformations for nodes [2, h7, b4] [parallel] and [2, H12, b6] [antiparallel] of the corresponding TN in Fig. 4), the interaction energies indicate that the antiparallel arrangement is favored. This is different from $\mathrm{A} \beta$ fibrils where only parallel $\beta$-sheets are found. Other preferred contacts in the dimers form between the $\mathrm{CHC}$ of one peptide and the C-terminal region of the other peptide. The fourth area with a certain, yet smaller probability of interpeptide contact is between the $\mathrm{CHC}$ regions of both peptides. However, these contacts are weaker than those between the two C-terminal regions, as the corresponding interaction energies are smaller in magnitude (SI Appendix, Fig. S10). The dissection of the interaction energies further reveals that attraction between the oppositely charged residues E22/D23 and K28 is involved in the association process, which is in agreement with previous findings (45).

The distance matrix of the membrane-adsorbed dimer looks similar to the one of the dimer in solution. However, the contact areas are more pronounced, indicating less structural diversity in the internal arrangement of the dimers. Second, the area without interpeptide contacts around residues D23 to K28 is larger. This applies to peptide 1 in particular and can be explained by the contacts that this peptide forms with the membrane instead. Third, the order of areas with the highest contact probability is different from those of the solution system. The shortest distances in the membrane-adsorbed dimer are observed between the $\mathrm{CHC}$ of peptide 1 and the C-terminal region of peptide 2 , followed by the contacts between both $\mathrm{CHC}$ regions. However, based on the secondary structure analysis, $\beta$-sheet formation between the two peptides is less likely and is largely limited to within peptide 


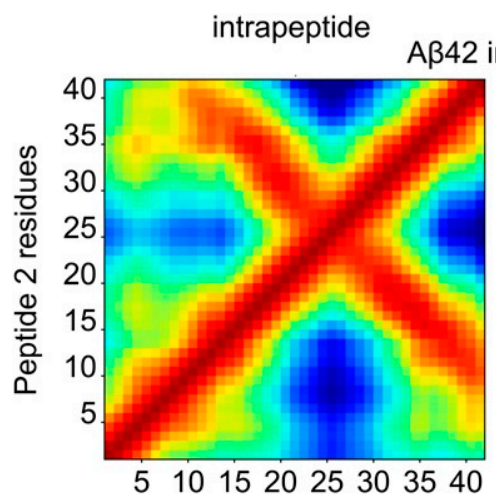

$A \beta 42$ in solution

interpeptide
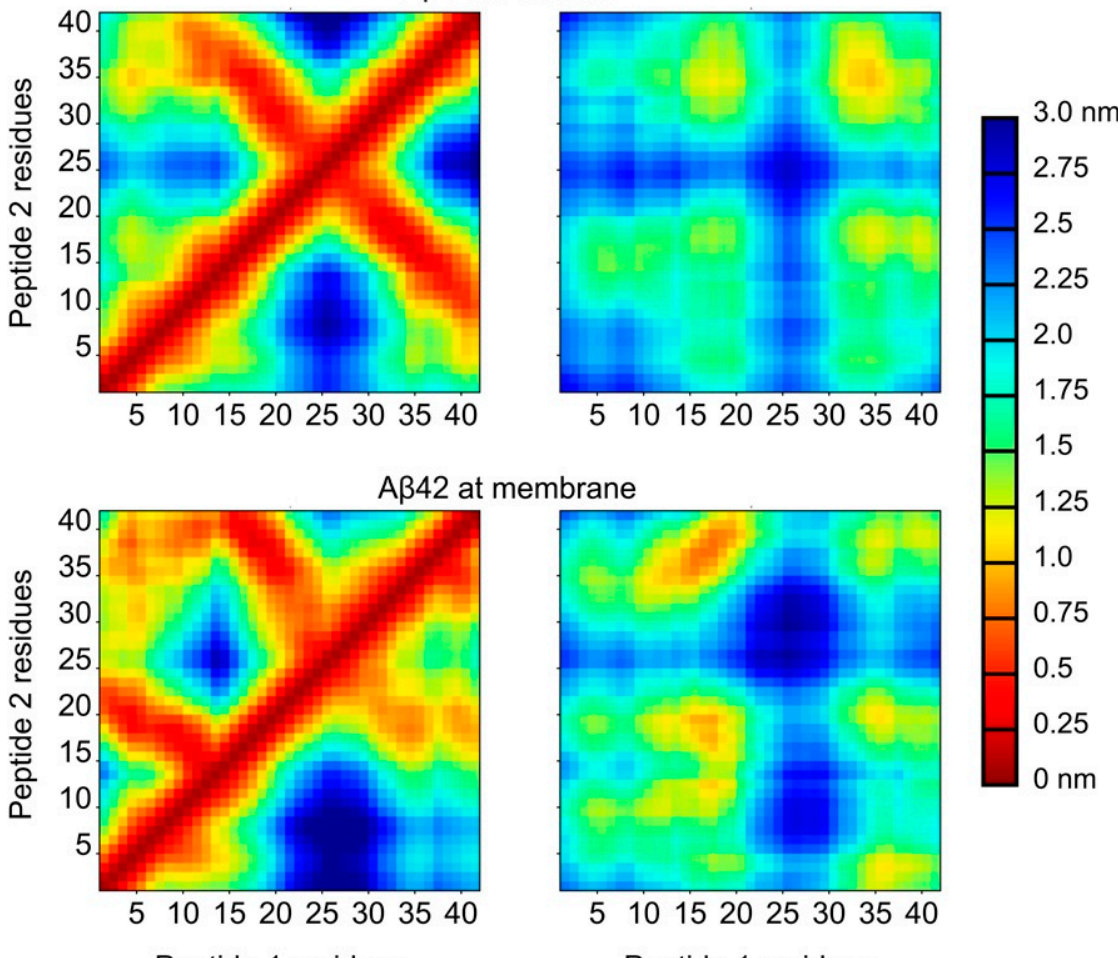

2.0

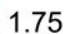

1.5

1.25

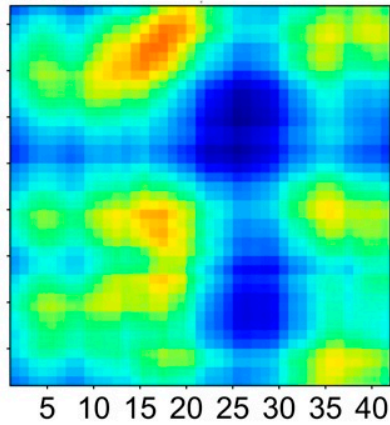

1.0

0.75

0.5

0.25

$0 \mathrm{~nm}$

Peptide 1 residues

Peptide 1 residues

Fig. 6. The distance matrices illustrating intra- and interpeptide contacts between residues for the dimer in the aqueous phase (Top) and in the presence of the neuronal membrane (Bottom). The intrapeptide contacts within peptide 1 are shown below the main diagonal and those within peptide 2 above it. The interpeptide contacts are shown for peptide 1 and peptide 2 composing the dimer. The color bar on the right indicates the average intra- and interresidue distances (in nanometers).

2. Contacts between the $\mathrm{C}$-terminal region of peptide 1 and the $\mathrm{N}$-terminal region of peptide 2 are also observed. As a result, the latter region exhibits an increased $\beta$-sheet propensity, which extends up to residue Y10 (Fig. 5A). It initially was assumed that the $\mathrm{N}$-terminal region of $\mathrm{A} \beta$ is always disordered. However, this was later refuted, first by simulations (SI Appendix, Fig. S9) and then by cryo-electron microscopy $(35,46)$. Contacts between the C-terminal regions are of less relevance for the membraneadsorbed dimer due to the competition between peptide-peptide and peptide-membrane interactions. The ranking of the interpeptide contact preferences is confirmed by the analysis of the interaction energies (SI Appendix, Fig. S10). Unlike in solution, attraction between E22/D23 and K28 does not play a role during the dimerization of $\mathrm{A} \beta$, which can be explained by the preference of $\mathrm{K} 28$ to associate with the membrane.

Reduced Global Motions but Increased Local Disorder in the Membrane-Adsorbed Dimer. To quantify the peptide dynamics, we calculated the $S^{2}$ order parameters to monitor the mobility of the N-H bond vectors of the peptide backbone along with the average global rotational correlation times, $\langle\tau\rangle$ (Fig. $5 B$ ). These quantities would be directly comparable to those determined by NMR spectroscopy, which, however, are not available yet. The global rotational dynamics of the $\mathrm{A} \beta 42$ dimer in solution occur on the low nanosecond time scale with $\langle\tau\rangle=20 \pm 10 \mathrm{~ns}$. The $S^{2}$ values reflect the different secondary structure propensities of the various residues. They are above $\approx 0.7$ for the residues in a $\beta$-conformation, while the more mobile turn region and neighboring residues ranging from E22 to A30 have $S^{2}$ values between 0.5 and 0.7 , and the disordered $\mathrm{N}$-terminal region has order parameters below 0.5 . The comparison to the $S^{2}$ values of the $\mathrm{A} \beta 40$ monomer confirm that the dimer in solution is considerably more folded, since for the monomer all $S^{2}$ values are below 0.4 (47). The global rotational dynamics of the $\mathrm{A} \beta 42$ dimer in the presence of the neuronal membrane are by a factor of 5 slower than in solution: $\langle\tau\rangle=108 \pm 30 \mathrm{~ns}$. Interestingly, the slower motion is accompanied by decreased order parameters compared to that seen in solution; the $S^{2}$ values range from 0.25 to 0.55 (and below 0.25 for the $\mathrm{N}$-terminal residues, similar to the situation in the solution dimer). The overall reduction in $S^{2}$ for the membrane-adsorbed dimer implies that the peptides are generally less folded than they are in solution, which agrees with the observed reduction in $\beta$-sheet and increase in random coil. Thus, a picture emerges where on the one hand the overall peptide dynamics are reduced due to the adsorption on the membrane, while at the same time the interactions with the membrane reduce the local peptide order as reflected by the $S^{2}$ values.

\section{Discussion}

In the present study, all-atom MD simulations on the microsecond time scale have been performed to elucidate the mechanism of $\mathrm{A} \beta 42$ dimerization in pure water and in the presence of a neuronal membrane. The consideration of a neuronal membrane consisting of six components (PC, PE, PS, CHOL, SM, and GM1) is a major step forward compared to previous simulation studies on $\mathrm{A} \beta$-membrane interactions, which included three lipid types or fewer. Dimerization was observed in the aqueous phase as well as at the neuronal membrane. However, the resulting dimer structures showed significant differences. Our simulations of $A \beta 42$ dimerization in solution revealed a coil-to- $\beta$ transition that is the first step along the amyloid 
aggregation pathway. The dimer conformations sampled in solution bear certain similarities to the $\beta$-sheets found in the Ushaped A $\beta 42$ amyloid fibrils. To our knowledge, a dimer structure with such a high $\beta$-sheet content and overall order has never been reported from all-atom MD simulations where the aggregation of $\mathrm{A} \beta$ progresses from disordered monomers into oligomers. We conclude that only the MD sampling of several microseconds and the use of a force field well suited to $\mathrm{A} \beta$ allow the random coil to $\beta$-sheet transition to be observed in a simulation (22). Thus, with these simulations we finally shed light on the structural transitions that might lead to nuclei enabling amyloid formation. Our future simulations will test whether the dimers that formed in solution here are indeed on pathway toward amyloid fibrils.

On the neuronal membrane, the dimer conformations are generally less ordered than in solution. The dimerization took place on the membrane, with one of the two peptides being preferentially adsorbed to the membrane and the other one associating with the already membrane-attached peptide without noteworthy interacting with the membrane itself. The directly adsorbed peptide in particular has a higher amount of random coil and less $\beta$-sheet. The membrane adsorption is mainly driven by electrostatic interactions between the charged $\mathrm{N}$-terminal residues of $\mathrm{A} \beta$ and the headgroups of PC, PE, and PS, in addition to hydrogen bonding between the sugar moieties of the GM1 lipids and A $\beta 42$ residues across its whole primary structure. GM1 is found to form clusters within the neuronal membrane, which are the preferable site for $\mathrm{A} \beta$ to bind to the membrane surface. This is in line with experimental results that revealed GM1 as part of a neuronal membrane to be the main interaction partner of $\mathrm{A} \beta$, whereas less binding was seen for SM and also PC (48). No insertion of the peptides into the hydrophobic region of the membrane was observed in our simulations. Instead, the interactions with the membrane stiffened both peptides, restricting their conformational diversity compared to the $\mathrm{A} \beta 42$ dimer simulated in the aqueous phase. Not only did the transition networks reveal a reduction in the number of conformational states, but also the correlation times of the $\mathrm{N}-\mathrm{H}$ bond vector motions indicated an impaired peptide motion. However, while adsorption was found to have profound effects on the $\mathrm{A} \beta 42$ dimer, the membrane was only marginally affected.

Our observations are in agreement with a large and diverse set of experimental results. Of special note is a study that analyzed the effects of glucose on $\mathrm{A} \beta 42$ aggregation (49). In this study, Kedia et al. (49) found that $\mathrm{A} \beta 42$ forms low-molecular-weight oligomers in the presence of sugars and that these oligomers do not adopt a $\beta$-sheet structure. This agrees with our observation that $\mathrm{A} \beta 42$ dimers that preferentially interact with the glycans of GM1 form fewer $\beta$-sheets than $\mathrm{A} \beta 42$ dimers that form in solution do. Moreover, another study revealed that $\mathrm{A} \beta$ oligomers that are present in the brain interstitial fluid are sequestered from that fluid by strongly binding to GM1, which also prevented the further aggregation of $\mathrm{A} \beta$ (48). We are aware of studies by Ikeda et al. (50) and Matsuzaki (51) that concluded that GM1 exhibits a strong Abeta fibril seeding potential following the formation of $\beta$-sheet-rich oligomers on GM1 clusters. However, these clusters are much larger than those formed in our simulations, as Ikeda et al. (50) and Matsuzaki (51) employed ganglioside-rich (>20 mol\% vs. the 4 mol\% used in our study) membranes, where GM1 forms an interconnected network of micrometer size yielding glycan platforms in liquid-ordered membranes. As elaborated by Hof and coworkers (52), the scenarios for membranes with high and low GM1 contents are not necessarily contradicting each other but rather complementary.

Another finding by the study of Kedia et al. (49) was that the unstructured $\mathrm{A} \beta 42$ oligomers that formed in the presence of glucose are able to interact with membrane bilayers. Their diffusion decreased by a factor of about 4 upon membrane adsorption, which agrees nicely with our observation that membrane interactions reduce the dynamics of the dimer. Moreover, no incorporation of the unstructured $\mathrm{A} \beta 42$ oligomers into the membrane was recorded (49), which also concurs with our findings. We conclude that, if a $\beta$-sheet structure should be required for membrane insertion of $\mathrm{A} \beta$ aggregates to occur, GM1 in the neuronal membrane has a neuroprotective effect as it could break the $\beta$-sheet structure in the $\mathrm{A} \beta$ dimer. This finding would be in agreement with the neuroprotective and neurogenerative effects reported for GM1 (53-55) and the conclusion that the neurotoxic activity of amyloid oligomers increases with their $\beta$ sheet content (8). On the other hand, Selkoe and coworkers (48) found that even though GM1 sequesters $\mathrm{A} \beta$ from the brain interstitial fluid, thereby inhibiting the aggregation of $\mathrm{A} \beta$, the binding of the peptide to GM1 alone mediates neurotoxic effects. This once more highlights that the interplay between $\mathrm{A} \beta$, its aggregation, and the neuronal membrane is far from trivial and despite the wealth of already published studies on that matter, further studies are needed to fully solve this puzzle.

\section{Materials and Methods}

Setup of the Simulated Systems. The systems modeled are composed of two A $\beta 42$ peptides, which were simulated in the aqueous phase and in the pres ence of the neuronal lipid membrane. The initial A $\beta 42$ structures were taken from the most populated clusters from a preceding 3- $\mu$ S MD simulation of monomeric $A \beta 42$ in solution. The neuronal membrane model composed of 152 PC, 96 PE, 20 PS, $80 \mathrm{CHOL}, 36 \mathrm{SM}$, and $16 \mathrm{GM} 1$ molecules was generated as symmetric lipid bilayer using the CHARMM-GUI interface (56).

The simulated membrane system also contained water layers above the upper and beneath the lower membrane leaflet, using the three-site transferable intermolecular potential (TIP3P) for modeling the water molecules, with sodium and chloride ions added at the physiological concentration of $150 \mathrm{mM}$. The two $\mathrm{A} \beta 42$ peptides were placed in the upper water layer at a distance of $\approx 2 \mathrm{~nm}$ from the equilibrated lipid bilayer surface and at a distance of $>1 \mathrm{~nm}$ between the closest atoms from the two peptides. All distances from the peptides to any of the simulation box edges were at least 1.2 $\mathrm{nm}$ to avoid interactions between the peptides and their periodic images. The total number of atoms in the modeled membrane system was $\approx 160,000$ atoms and the box size was about $9.6 \times 9.6 \times 13.6 \mathrm{~nm}^{3}$. The setup of the system in the aqueous phase was similar, but without a lipid bilayer, resulting in a system size of about $9.2 \times 9.2 \times 6.5 \mathrm{~nm}^{3}$, and contained $\approx 54,760$ atoms. This amounts to peptide concentrations of 4 and $6 \mathrm{mM}$, respectively. This is two to three orders of magnitude higher than the concentrations used in corresponding in vitro experiments. However, it is beyond our computational capabilities to model $\mu \mathrm{M}$ peptide concentrations at the atomistic level. Moreover, simulations at such low concentrations would most of the time simulate only the diffusion of monomeric peptides (57). We therefore aim to model the oligomerization of $A \beta$ in a stepwise fashion $(57,58)$ starting here with simulations of dimers.

MD Simulation Conditions. The all-atom MD simulations were performed using GROMACS/2018.2 (59) along with the CHARMM36m force field for $A \beta 42$ (60) and Charmm36 for the lipids (61). Each system was first energy minimized using the steepest-descent algorithm to remove atomic clashes. This was followed by equilibration in the canonical ensemble where a temperature of $310 \mathrm{~K}$ was regulated with the velocity-rescale thermostat (62) Next, the system was equilibrated under isobaric-isothermic conditions to obtain a pressure of 1.0 bar, where the pressure was regulated using a semi-isotropic Parrinello-Rahman pressure coupling scheme (63). Periodic boundary conditions were set in all directions. Both the van der Waals and Coulomb force cutoffs were set to $1.2 \mathrm{~nm}$ in real space. The particle mesh Ewald (PME) method was applied for calculating the electrostatic interactions. Before we studied the interaction of $A \beta 42$ with the neuronal membrane, we equilibrated the membrane without peptides being present for $1 \mu \mathrm{s}$. For A $\beta 42$ dimer systems, an initial simulation was run for $2 \mu \mathrm{s}$ from which different snapshots were randomly selected and used as starting structures for the next $5 \times 2-\mu$ s simulations. For the subsequent analysis, we combined the data from the six independent simulations and derived the results presented in this study.

Analysis of the Lipid Bilayer Properties. For the determination of the order parameter of the lipid acyl chains, $\mathrm{S}_{\mathrm{CH}}$, one uses the $\mathrm{C}-\mathrm{H}$ bond vectors 
present in the lipid tails and calculates the orientation of these vectors with respect to the bilayer normal (the $z$ axis) using

$$
S_{\mathrm{CH}}=\frac{\left\langle 3 \cos ^{2} \theta-1\right\rangle}{2}
$$

where $\theta$ is the angle between the $\mathrm{C}-\mathrm{H}$ bond vector and the bilayer normal. The angular brackets indicate the ensemble average. This calculation was accomplished with a Python script available at https://github. com/NMRLipids/MATCH (64).

The mass density profiles along the bilayer normal were calculated using the "gmx density" tool. The distance between the peaks of the total density gives an estimate of the bilayer thickness. Furthermore, the bilayer thickness was calculated as the $z$-position difference between the P atoms of the lipid headgroups in the upper and lower leaflets using the "gmx distance" tool. The RDF provides information about the probability of finding a particle at a certain distance from another particle. We calculated the radial distribution functions of different lipid pairs in two dimensions (the $x y$ plane) using the "gmx rdf" tool. The hydrogen bonds between different lipid pairs were determined using "gmx hbond." A hydrogen bond was recorded when the angle between the donor and acceptor bonded hydrogen was between 150 and $180^{\circ}$ and the distance between the two atoms was within $0.35 \mathrm{~nm}$.

Analysis of $\mathbf{A} \boldsymbol{\beta} 42$ Properties. The secondary structure of each $\mathrm{A} \beta 42$ residue was determined using the "define secondary structure program" (DSSP) (65) invoked via the GROMACS tool "do dssp." To facilitate a clear representation, the data of similar secondary structures are grouped together: $\beta$-strand and $\beta$-bridge are combined as $\beta$-sheet and $\beta$-turn and bend as turn/bend; and the helix includes $\alpha, \pi$, and $3_{10}$-helices.

For the calculation of the $S^{2}$ order parameter we used the MOPS ${ }^{2}$ (Molecular Order Parameter $S^{2}$ ) software developed in ref. 66 to calculate $S^{2}$ from the $\mathrm{N}-\mathrm{H}$ bond vector autocorrelation function. To facilitate the calculation, each trajectory was divided into subtrajectories of $t_{\text {sub }}=100 \mathrm{~ns}$ length. For each of the subtrajectories the $S^{2}$ values and the rotational correlation times, $\tau$, were calculated and subsequently averaged over all subtrajectories. The rotational correlation times were further averaged over all residues and both peptides, denoted as $\langle\tau\rangle$, whereas $S^{2}$ is provided per residue and peptide. Since $\langle\tau\rangle$ for the membrane system is in the same range as $t_{\text {sub }}$, we checked on the convergence for the $S^{2}$ calculation in this case (SI Appendix, Fig. S11).

Transition Networks. For the generation of the TNs to characterize the assembly of peptides into dimers we used the ATRANET (Automated Transition Network) software (https://github.com/strodel-group/ATRANET) (27). It defines the oligomerization state by a number of descriptors, depending on the properties of interest. In our case, three descriptors are used: The first one is the oligomer size, which can be 1 in the case of monomer or 2 in the case of a dimer. To define a dimer, the minimum distance between any atom of peptide 1 and any atom of peptide 2 along with the requirement of this distance to be within $0.45 \mathrm{~nm}$ was used. The second descriptor, the number of hydrophobic contacts between both peptides, counts the possible interpeptide atom pairs formed between the hydrophobic amino acids of $A \beta 42$ that are within a certain cutoff (also $0.45 \mathrm{~nm}$ ). The third descriptor is the number of residues in $\beta$-strand conformation, which is evaluated using DSSP and averaged over both peptides. Feeding these descriptors to ATRANET leads to a transition matrix that can be visualized using Gephi (67). Snapshots of the representative structures from the transition network were rendered using the visual molecular dynamics (VMD) program (68).

Calculation of $\mathbf{A} \boldsymbol{\beta} 42-\mathbf{B}$ ilayer Interactions. The peptide-lipid interactions were analyzed by calculating the interaction energy between each $A \beta 42$ residue and the headgroup of each lipid component using "gmx energy." The "gmx mindist" program was employed to determine the number of contacts between each $A \beta 42$ residue and each lipid component in the neuronal membrane. A contact was recorded when the distance between any two nonhydrogen atoms from a residue and a lipid was within $0.5 \mathrm{~nm}$. The $\mathrm{H}$-bond propensity was determined by the number of times an $\mathrm{H}$ bond was formed between hydrogen bond donating and accepting atoms in lipid pairs.

Data Availability. The MD trajectories and the analysis scripts are available at Mendeley Data, https://data.mendeley.com/datasets/92mkp4pk86. All data resulting from the analysis of this raw data is shown in the main text or SI Appendix.

ACKNOWLEDGMENTS. H.F., M.K., A.S.-A., and B.S. acknowledge funding for this project from the Palestinian-German Science Bridge financed by the German Federal Ministry of Education and Research. B.S. received funding for this project from the Deutsche Forschungsgemeinschaft (German Research Foundation, https://www.dfg.de/) through Grant 267205415 (CRC 1208 , Project A07). M.C.O. received funding for this project from the European Union's Horizon 2020 research and innovation programme under the Marie Skłodowska-Curie grant and it is cofinanced by the South Moravian Region under Grant 665860. This article reflects only the authors' view and the European Union is not responsible for any use that may be made of the information it contains. This research was supported by the European Union and the Hungarian State, cofinanced by the European Regional Development Fund in the framework of the GINOP-2.3.4-15-2016-00004 project, aimed to promote the cooperation between the higher education and the industry. M.C.O. and B.S. gratefully acknowledge the Gauss Centre for Supercomputing (GCS) e.V. (www.gauss-centre.eu) for funding this project by providing computing time on the GCS Supercomputer SuperMUC-NG at Leibniz Supercomputing Centre (https://www.Irz.de/). H.F., M.K., and B.S. gratefully acknowledge the computing time granted through Jülich Aachen Research Alliance - High Performance Computing (Projects JICS6C and AMYLOID-MSM) on the supercomputer Jureca at Forschungszentrum Jülich.
1. S. H. Barage, K.D. Sonawane, Amyloid cascade hypothesis: Pathogenesis and therapeutic strategies in Alzheimer's disease. Neuropeptides 52, 1-18 (2015).

2. C. A. McLean et al., Soluble pool of Abeta amyloid as a determinant of severity of neurodegeneration in Alzheimer's disease. Ann. Neurol. 46, 860-866 (1999).

3. M. D. Kirkitadze, G. Bitan, D. B. Teplow, Paradigm shifts in Alzheimer's disease and other neurodegenerative disorders: The emerging role of oligomeric assemblies. J. Neurosci. Res. 69, 567-577 (2002).

4. K. Broersen, F. Rousseau, J. Schymkowitz, The culprit behind amyloid beta peptide related neurotoxicity in Alzheimer's disease: Oligomer size or conformation? Alzheimers Res. Ther. 2, 12 (2010).

5. A. Müller-Schiffmann et al., Amyloid- $\beta$ dimers in the absence of plaque pathology impair learning and synaptic plasticity. Brain 139, 509-525 (2016).

6. G. Brinkmalm et al., Identification of neurotoxic cross-linked amyloid- $\beta$ dimers in the Alzheimer's brain. Brain 142, 1441-1457 (2019).

7. G. Bitan, D. B. Teplow, Rapid photochemical cross-linking-A new tool for studies of metastable, amyloidogenic protein assemblies. Acc. Chem. Res. 37, 357-364 (2004).

8. K. Ono, M. M. Condron, D. B. Teplow, Structure-neurotoxicity relationships of amyloid beta-protein oligomers. Proc. Natl. Acad. Sci. U.S.A. 106, 14745-14750 (2009).

9. B. O'Nuallain et al., Amyloid beta-protein dimers rapidly form stable synaptotoxic protofibrils. J. Neurosci. 30, 14411-14419 (2010).

10. A. Vázquez de la Torre et al., Direct evidence of the presence of cross-linked a $\beta$ dimers in the brains of Alzheimer's disease patients. Anal. Chem. 90, 4552-4560 (2018).

11. F. Castello et al., Two-step amyloid aggregation: Sequential lag phase intermediates. Sci. Rep. 7, 40065 (2017).

12. J. Yang et al., Direct observation of oligomerization by single molecule fluorescence reveals a multistep aggregation mechanism for the yeast prion protein ure2. J. Am. Chem. Soc. 140, 2493-2503 (2018).
13. J. Nasica-Labouze et al., Amyloid $\beta$ protein and Alzheimer's disease: When computer simulations complement experimental studies. Chem. Rev. 115, 3518-3563 (2015).

14. P. H. Nguyen et al., Amyloid oligomers: A joint experimental/computational perspective on Alzheimer's disease, Parkinson's disease, type II diabetes, and amyotrophic lateral sclerosis. Chem. Rev. 121, 2545-2647 (2021).

15. S. J. C. Lee, E. Nam, H. J. Lee, M. G. Savelieff, M. H. Lim, Towards an understanding of amyloid- $\beta$ oligomers: Characterization, toxicity mechanisms, and inhibitors. Chem. Soc. Rev. 46, 310-323 (2017).

16. M. C. Owen et al., Effects of in vivo conditions on amyloid aggregation. Chem. Soc. Rev. 48, 3946-3996 (2019).

17. Z. Korade, A. K. Kenworthy, Lipid rafts, cholesterol, and the brain. Neuropharmacology 55, 1265-1273 (2008).

18. E. Posse de Chaves, S. Sipione, Sphingolipids and gangliosides of the nervous system in membrane function and dysfunction. FEBS Lett. 584, 1748-1759 (2010).

19. Y. C. Kao, P. C. Ho, Y. K. Tu, I. M. Jou, K. J. Tsai, Lipids and Alzheimer's disease. Int. J. Mol. Sci. 21, 1505 (2020).

20. A. Paul, S. Samantray, M. Anteghini, M. Khaled, B. Strodel, Thermodynamics and kinetics of the amyloid- $\beta$ peptide revealed by Markov state models based on MD data in agreement with experiment. Chem. Sci. (Camb.) 12, 6652-6669 (2021).

21. S. Samantray, F. Yin, B. Kav, B. Strodel, Different force fields give rise to different amyloid aggregation pathways in molecular dynamics simulations. J. Chem. Inf. Model. 60, 6462-6475 (2020).

22. B. Strodel, Amyloid aggregation simulations: Challenges, advances and perspectives. Curr. Opin. Struct. Biol. 67, 145-152 (2021).

23. H. Koldsø, D. Shorthouse, J. Hélie, M. S. Sansom, Lipid clustering correlates with membrane curvature as revealed by molecular simulations of complex lipid bilayers. PLOS Comput. Biol. 10, e1003911 (2014).

24. H. I. Ingólfsson et al., Computational lipidomics of the neuronal plasma membrane. Biophys. J. 113, 2271-2280 (2017). 
25. B. Barz, D. J. Wales, B. Strodel, A kinetic approach to the sequence-aggregation relationship in disease-related protein assembly. J. Phys. Chem. B 118, 1003-1011 (2014).

26. B. Barz, Q. Liao, B. Strodel, Pathways of amyloid- $\beta$ aggregation depend on oligomer shape. J. Am. Chem. Soc. 140, 319-327 (2018).

27. A. M. Illig, B. Strodel, Performance of Markov state models and transition networks on characterizing amyloid aggregation pathways from MD data. J. Chem. Theory Comput. 16, 7825-7839 (2020).

28. J. P. Jämbeck, A. P. Lyubartsev, An extension and further validation of an all-atomistic force field for biological membranes. J. Chem. Theory Comput. 8, 2938-2948 (2012).

29. I. Ermilova, A. P. Lyubartsev, Extension of the slipids force field to polyunsaturated lipids. J. Phys. Chem. B 120, 12826-12842 (2016).

30. J. Egawa, M. L. Pearn, B. P. Lemkuil, P. M. Patel, B. P. Head, Membrane lipid rafts and neurobiology: Age-related changes in membrane lipids and loss of neuronal function. J. Physiol. 594, 4565-4579 (2016).

31. V. Pata, N. Dan, Effect of membrane characteristics on phase separation and domain formation in cholesterol-lipid mixtures. Biophys. J. 88, 916-924 (2005).

32. H. Ohvo-Rekilä, B. Ramstedt, P. Leppimäki, J. P. Slotte, Cholesterol interactions with phospholipids in membranes. Prog. Lipid Res. 41, 66-97 (2002).

33. M. Coles, W. Bicknell, A. A. Watson, D. P. Fairlie, D. J. Craik, Solution structure of amyloid beta-peptide(1-40) in a water-micelle environment. Is the membrane-spanning domain where we think it is? Biochemistry 37, 11064-11077 (1998).

34. N. Miyashita, J. E. Straub, D. Thirumalai, Structures of $\beta$-amyloid peptide $1-40,1-$ 42, and 1-55 - the 672-726 fragment of app - in a membrane environment with implications for interactions with $\gamma$-secretase. J. Am. Chem. Soc. 131, 17843-17852 (2009).

35. B. Strodel, J. W. L. Lee, C. S. Whittleston, D. J. Wales, Transmembrane structures for Alzheimer's A $\beta$ (1-42) oligomers. J. Am. Chem. Soc. 132, 13300-13312 (2010).

36. L. Nagel-Steger, M. C. Owen, B. Strodel, An account of amyloid oligomers: Facts and figures obtained from experiments and simulations. ChemBioChem 17, 657-676 (2016).

37. T. Kakeshpour et al., A lowly populated, transient $\beta$-sheet structure in monomeric a $\beta$ 1-42 identified by multinuclear NMR of chemical denaturation. Biophys. Chem. 270, 106531 (2020)

38. B. Urbanc et al., Molecular dynamics simulation of amyloid beta dimer formation. Biophys. J. 87, 2310-2321 (2004).

39. S. Côté, R. Laghaei, P. Derreumaux, N. Mousseau, Distinct dimerization for various alloforms of the amyloid-beta protein: $A \beta 1-40, \beta 1-42$, and $\beta 1-40(d 23 n)$. J. Phys. Chem. B 116, 4043-4055 (2012).

40. V. H. Man, P. H. Nguyen, P. Derreumaux, High-resolution structures of the amyloid- $\beta-$ 42 dimers from the comparison of four atomistic force fields. J. Phys. Chem. B 121, 5977-5987 (2017).

41. B. Mehrazma, A. Rauk, Exploring amyloid- $\beta$ dimer structure using molecular dynamics simulations. J. Phys. Chem. A 123, 4658-4670 (2019).

42. S. Rauscher et al., Structural ensembles of intrinsically disordered proteins depend strongly on force field: A comparison to experiment. J. Chem. Theory Comput. 11, 5513-5524 (2015).

43. T. Lührs et al., 3D structure of Alzheimer's amyloid- $\beta$ (1-42) fibrils. Proc. Natl. Acad. Sci. U.S.A. 102, 17342-17347 (2005).

44. A. K. Paravastu, R. D. Leapman, W. M. Yau, R. Tycko, Molecular structural basis for polymorphism in Alzheimer's beta-amyloid fibrils. Proc. Natl. Acad. Sci. U.S.A. 105, 18349-18354 (2008).

45. B. Tarus, J. E. Straub, D. Thirumalai, Structures and free-energy landscapes of the wild type and mutants of the $A \beta(21-30)$ peptide are determined by an interplay between intrapeptide electrostatic and hydrophobic interactions. J. Mol. Biol. 379, 815-829 (2008).
46. L. Gremer et al., Fibril structure of amyloid- $\beta(1-42)$ by cryo-electron microscopy. Science 358, 116-119 (2017).

47. N. Rezaei-Ghaleh, G. Parigi, M. Zweckstetter, Reorientational dynamics of amyloid- $\beta$ from NMR spin relaxation and molecular simulation. J. Phys. Chem. Lett. 10, 33693375 (2019).

48. S. Hong et al., Soluble $A \beta$ oligomers are rapidly sequestered from brain ISF in vivo and bind GM1 ganglioside on cellular membranes. Neuron 82, 308-319 (2014).

49. N. Kedia, M. Almisry, J. Bieschke, Glucose directs amyloid- $\beta$ into membrane-active oligomers. Phys. Chem. Chem. Phys. 19, 18036-18046 (2017).

50. K. Ikeda, T. Yamaguchi, S. Fukunaga, M. Hoshino, K. Matsuzaki, Mechanism of amyloid $\beta$-protein aggregation mediated by GM1 ganglioside clusters. Biochemistry $\mathbf{5 0}$ 6433-6440 (2011)

51. K Matsuzaki, Formation of toxic amyloid fibrils by amyloid $\beta$ protein on ganglioside clusters. Int. J. Alzheimers Dis. 2011, 956104 (2011).

52. M. Cebecauer, M. Hof, M. Amaro, Impact of GM1 on membrane-mediated aggregation/oligomerization of $\beta$-amyloid: Unifying view. Biophys. J. 113, 1194-1199 (2017).

53. T. V. Sokolova, I. O. Zakharova, V. V. Furaev, M. P. Rychkova, N. F. Avrova, Neuroprotective effect of ganglioside GM1 on the cytotoxic action of hydrogen peroxide and amyloid beta-peptide in PC12 cells. Neurochem. Res. 32, 1302-1313 (2007).

54. F. Kreutz et al., Amyloid- $\beta$ induced toxicity involves ganglioside expression and is sensitive to GM1 neuroprotective action. Neurochem. Int. 59, 648-655 (2011).

55. R. Yang et al., Monosialoanglioside improves memory deficits and relieves oxidative stress in the hippocampus of rat model of Alzheimer's disease. Neurol. Sci. 34, 14471451 (2013).

56. J. Lee et al., CHARMM-GUI input generator for NAMD, GROMACS, AMBER, openMM, and CHARMM/openMM simulations using the CHARMM36 additive force field. $J$. Chem. Theory Comput. 12, 405-413 (2016).

57. M. Carballo-Pacheco, B. Strodel, Advances in the simulation of protein aggregation at the atomistic scale. J. Phys. Chem. B 120, 2991-2999 (2016).

58. U. Sengupta, M. Carballo-Pacheco, B. Strodel, Automated Markov state models for molecular dynamics simulations of aggregation and self-assembly. J. Chem. Phys. 150, 115101 (2019).

59. M. J. Abraham et al., GROMACS: High performance molecular simulations through multi-level parallelism from laptops to supercomputers. SoftwareX 1, 19-25 (2015).

60. J. Huang et al., CHARMM $36 \mathrm{~m}$ : An improved force field for folded and intrinsically disordered proteins. Nat. Methods 14, 71-73 (2017)

61. J. B. Klauda et al., Update of the CHARMM all-atom additive force field for lipids: Validation on six lipid types. J. Phys. Chem. B 114, 7830-7843 (2010).

62. G. Bussi, D. Donadio, M. Parrinello, Canonical sampling through velocity rescaling. J. Chem. Phys. 126, 014101 (2007)

63. H. J. Berendsen, J. Postma, W. F. van Gunsteren, A. DiNola, J. R. Haak, Molecula dynamics with coupling to an external bath. J. Chem. Phys. 81, 3684-3690 (1984).

64. H. Antila et al., Headgroup structure and cation binding in phosphatidylserine lipid bilayers. J. Phys. Chem. B 123, 9066-9079 (2019).

65. Y. Zhang, C. Sagui, Secondary structure assignment for conformationally irregular peptides: Comparison between DSSP, STRIDE and KAKSI. J. Mol. Graph. Model. 55, 72-84 (2015).

66. C. Möckel et al., Integrated NMR, fluorescence, and molecular dynamics benchmark study of protein mechanics and hydrodynamics. J. Phys. Chem. B 123, 1453-1480 (2019).

67. M. Bastian et al., Gephi: An open source software for exploring and manipulating networks. Proc. Third Int. ICWSM Conf. 8, 361-362 (2009).

68. W. Humphrey, A. Dalke, K. Schulten, VMD: Visual molecular dynamics. J. Mol. Graph 14, 33-38 (1996) 